\title{
On the propagation of a conceptual error concerning hypercycles and cooperation
}

Eörs Szathmáry ${ }^{1,2,3}$

\begin{abstract}
The hypercycle is a system of replicators, whose members are auto- and cross-catalytic: replication of each member is catalyzed by at least one other member of the system. Therefore, the kinetics of growth of every member is at least second order. In ecology such systems are called mutualistic whose members are cooperating with each other. The dynamics of such systems are described broadly by the replicator equation. In chemistry hypercycles are often confused with collectively autocatalytic systems in which the members catalyze each other's formation rather than replication (growth being therefore first-order). Examples of this confusion abound in the literature. The trouble is that such category errors mistakenly imply that the available theories of hypercycles and cooperation are applicable, although in fact they are not. Cooperation in population biology means a higher-order interaction among agents with (at least the capacity of) multiplication. From the point of evolution, what matters is the genetic effects on the cooperative act. As systems chemistry has one of its roots in the theoretical biology, insights from this field ought to be respected even by experimentalists, let alone theoreticians.
\end{abstract}

Keywords: Autocatalysis, Hypercycle, Collectively autocatalytic sets, Replicator dynamics, Cooperation Category error

\section{Background}

The molecular hypercycle as proposed by Eigen [1] and elaborated by Eigen and Schuster [2] is a system in which autocatalytic replicators also heterocatalytically aid each other's replication so that replication of each member is catalyzed by at least one other member. If we have two replicators then the simplest stoichiometric scheme is:

$$
\begin{aligned}
& \mathrm{I}_{1}+\mathrm{I}_{2}+\mathrm{R}_{1} \stackrel{k_{1}}{\rightarrow} 2 \mathrm{I}_{1}+\mathrm{I}_{2} \\
& \mathrm{I}_{2}+\mathrm{I}_{1}+\mathrm{R}_{2} \stackrel{k_{2}}{\rightarrow} 2 \mathrm{I}_{2}+\mathrm{I}_{1},
\end{aligned}
$$

where $\mathrm{I}_{i}$ are the replicators and $\mathrm{R}_{i}$ are the adequate resources, of which the dynamics is usually neglected, by absorbing their concentrations into the rate constants $k_{i}$. It is readily obvious that the kinetic equations crucially include growth terms involving $k_{i}\left[\mathrm{I}_{i}\right]$ $\left[\mathrm{I}_{j}\right]$, hence the term second-order autocatalysis. Apart

\footnotetext{
Correspondence: szathmary.eors@gmail.com

${ }^{1}$ Parmenides Center for the Conceptual Foundations of Science, Kirchplatz 1 Pullach, Munich D-82049, Germany

${ }^{2}$ Faculty of Biology, Ludwig Maximilians University Munich, Munich, Germany Full list of author information is available at the end of the article
}

from the relevance (or not) of such systems in early molecular evolution, they have triggered considerable, respectable mathematical work. It has been realized that the kinetic model of the hypercycle can be regarded as a special case of the replicator equation, of which the other special cases include Lotka-Volterra systems (needing a barycentric transformation), Fishertype genetic selection and evolutionary game dynamics [3]. One has to pay special attention to the issue of cooperation here. The hypercycle is an example of molecular mutualists from an ecological point of view: it is analogous to the case in which Species 1 helps the reproduction of Species 2 that helps the reproduction of Species 1. (Similar cases can be defined within species). The hypercycle idea is so clear and simple that it seems impossible to misunderstand it. Yet, very unfortunately, chemists excel in such misunderstanding. One might say that this is semantic quibble, but it is not, for the following reason. IF one thinks that one is dealing with a cooperative/hypercyclic system, THEN one readily assumes that the THEORY developed for such systems [3] is applicable. Thus if one makes a category 
error of this kind, adverse consequences follow. To put it bluntly, one does not know what one is talking about.

\section{Analysis}

Let us see the examples. The first case when I saw this happen was in two papers in which coupled cyclic biochemical systems were treated and called hypercycles, although the case did not include a single replicator or autocatalysis of any kind [4,5]. I analyzed the case shortly afterwards under the title "a hypercyclic illusion" [6], naively thinking that the issue will be put to rest. Not so. The next case is the collectively autocatalytic peptide sets of Ghadiri [7], where the paper had "emergence of symbiosis" in its title, on the assumption that the authors had produced a hypercyclic network. In its simplest form we are dealing with a system that looks like this:

$$
\begin{aligned}
& \mathrm{I}_{2}+\mathrm{R}_{1} \stackrel{k_{1}}{\rightarrow} \mathrm{I}_{1}+\mathrm{I}_{2} \\
& \mathrm{I}_{1}+\mathrm{R}_{2} \stackrel{k_{2}}{\rightarrow} \mathrm{I}_{2}+\mathrm{I}_{1} .
\end{aligned}
$$

It should be obvious that $I_{1}$ catalysis the formation rather than the replication of $\mathrm{I}_{2}$ and vice versa. This is not a hypercycle, neither a case of cooperation as defined in the classical theory [8] of evolutionary biology. It is (importantly) a case of a collectively autocatalytic set to which Stu Kauffman [9] has paid special attention throughout the

\section{Table 1 Quotes confusing cross-catalysis with hypercyclic coupling} "They also showed that these types of peptides can symbiotically replicate each other through a hypercycle, with autocatalytic rate ([17], p. 420)
enhancements of several thousands over the background reactions"

"Given the proximity of the Joyce and Ghadiri labs at the Scripps Research Institute, perhaps such crossreplicating nucleic acid and ([18], p. 14) peptide hypercycles are already being born, either planned or unplanned"

"A system containing two self-replicating coil peptides, R1 and R2, exhibited a form of symbiosis in which each sequence was able to catalyse the formation of the other.... the two replicators are not mutually exclusive, and a hypercyclic network is evident"

"Since this initial breakthrough, chemists have devised increasingly complex cross-catalytic systems and are currently working on hypercycles, which are crosscomplementary self-replicating systems with more than twotemplate molecules. In these systems template $A$ catalyzes the formation of template $B$, which catalyzes the formation of template $C$, etc., until the final template catalyzes the formation of template A to complete the cycle"

"self replicating peptides have been designed with many of the fundamental properties of living systems, including dynamic error correction, chiroselectivity and hypercycle catalytic networks."

"Diagram of a hypercycle in which two autocatalytic replicators, $\mathbf{R} 1(\mathbf{E} / \mathbf{N} 1)$ and $\mathbf{R 2}(\mathbf{E} / \mathbf{N 2})$, are connected to each other via a crosscatalytic network. $\mathbf{R} \mathbf{1}$ catalyzes the formation of $\mathbf{R} \mathbf{2}$, as well as of itself, and vice versa, which prevents one replicator from overwhelming the other and enables the two to reproduce as a single coherent unit.... A hypercycle is a collective of two or more self-replicating species interlinked through a cyclic catalytic network. Lee et al. have designed a simple form of a hypercyclic network from two peptide replicators.... This mode of catalytic coupling prevents one replicator from overwhelming the other and unifies two otherwise competitive species into a single cooperative reproducing entity."

"Given that discrete peptides and organic compounds have been shown to be capable of self-replication, the emergence of novel properties from combinatorial libraries of such replicators can be readily imagined. As an example, Ghadiri and coworkers have shown the emergence of coupled hypercycles of replicating peptides"

"This led to a mutualistic, interconnected cycle in which valine-substituted replicators could make isoleucine substituted replicators, and vice versa.... Peptide hypercycles. Green represents a peptide with, say, valine in a key position, while red represents a peptide with an isoleucine in the same position. Unlike nucleic acids, they are capable of efficient cross-replication"

"For a peptide replicator, templating is less exact, so the formation of a mutant template is common. The mutant template can catalyze formation of mutant progeny or parental progeny, and the two species form a mutualistic network... The replication hypercycle consists of two intertwined polymerization and recombination cycles. In one cycle, polymerization of the short RNA fragments comprising the polymerase and recombinase occurs through primer extension and dissociation of sense/antisense strands. In the other cycle, the reconstituted recombinase stitches the RNA fragments. Recombination is directed by internal guide sequences, forming longer, more complex ribozymes"

"There must have been a different, better path to origins, perhaps via the evolution of a hypercyclic network rather than a single ribozyme"

"Hypercycles are unique in that individual reproductive cycles are connected by functional linkages. Within the context of the proposed hypercycle, short RNA fragments are replicated by the polymerase and then stitched together by the Azoarcus ribozyme. This obviates the requirement that a complex replicase spontaneously emerge from simple precursors as well as the need for the polymerase to act on structured templates. Thus, while neither species can efficiently self-replicate, each can survive in the context of the hypercycle. Envisioning this mutualistic network begs the problem of how to confine benefits to the two members of the network."

"The three-membered cycle shown here resembles a hypercycle as envisioned previously but without hyperbolic growth."

"Vaidya et al. show that variants of such RNA fragments can assemble and act on one another to form cooperative self-assembly cycles very much like the proposed hypercycles, in which ribozyme 1 aids assembly of ribozyme 2; 2 aids 3; and 3 aids 1" 
years. Such sets have been realized, emphatically without a conceptual flow in interpretation, by von Kiedrowski $[10,11]$. Note that in system (3-4) $I_{1}$ and $I_{2}$ are not replicators, only the set $\left\{\mathrm{I}_{1}, \mathrm{I}_{2}\right\}$ can be regarded as an ensemble replicator [12]. Fortunately, the claim about such systems being hypercyclic has been withdrawn [13], but unfortunately many people have not taken notice: Table 1 presents examples of spectacular conceptual error propagation. The latest similar case is that of a collectively autocatalytic set of RNA ligases [14], for which even the associated News and Views [15] regarded the presented system hypercyclic. This is completely wrong I am afraid, for the reasons explained above. (Note that some of the ligases involved can directly be autocatalytic, but these reactions run in parallel to the formation aided by crosscatalysis.) Unfortunately, similar errors can propagate into literature meant to inform the wider public about key developments in evolution [16].

In order to help the reader feel in another way the magnitude of the error, I daresay the cited mistakes reach the level of confusing momentum $(m v)$ with kinetic energy $\left(m v^{2} / 2\right)$ in mechanics: the first expression is linear, while the second is quadratic in velocity. There are different theorems for momentum and energy which cannot be confused, even though they are related in the sense that both are dealing with motion.

Finally, I present a case where again the system has been called a hypercycle without hypercyclic coupling.
Curiously, a predator-prey like interaction is involved, however (Figure 1). This is an interesting system that has been presented as a possible solution of the problem of early RNA replication [24]. In brief, a polymerase helps the replication of RNA oligomers and a ligase helps the formation of itself as well as of the replicase out of these oligomers. This system is exciting, but what is it from the point of view of ecological/evolutionary interactions, if it is not hypercyclic? Figure 1 attempts to give an answer. The closest (but still limited) analogy I can think of is that of two tropic levels and sterile and worker castes in a social insect. The sterile caste does the farming of fungi on which the larvae feed whose fate to become sterile or fertile depends on diet. (I can assure fellow systems chemists that such cases exist). Again, the system is collectively autocatalytic but there is no direct mutual catalysis of replication (but there are other interactions usually not considered in the theory of autocatalytic sets). Polymerase helps the replication of the oligomers but the latter contribute stoichiometrically rather than catalytically to the formation of polymerase and the autocatalysis of the ligase. A crucial difference between the two systems is that, in contrast to the social insect case, polymerase and ligase are not genetically related, so the polymerase does not forgo its own direct replication by kin selection [25] to aid the replication of the ligase by producing food. Apart from this, is there molecular cooperation here in a biologically meaningful
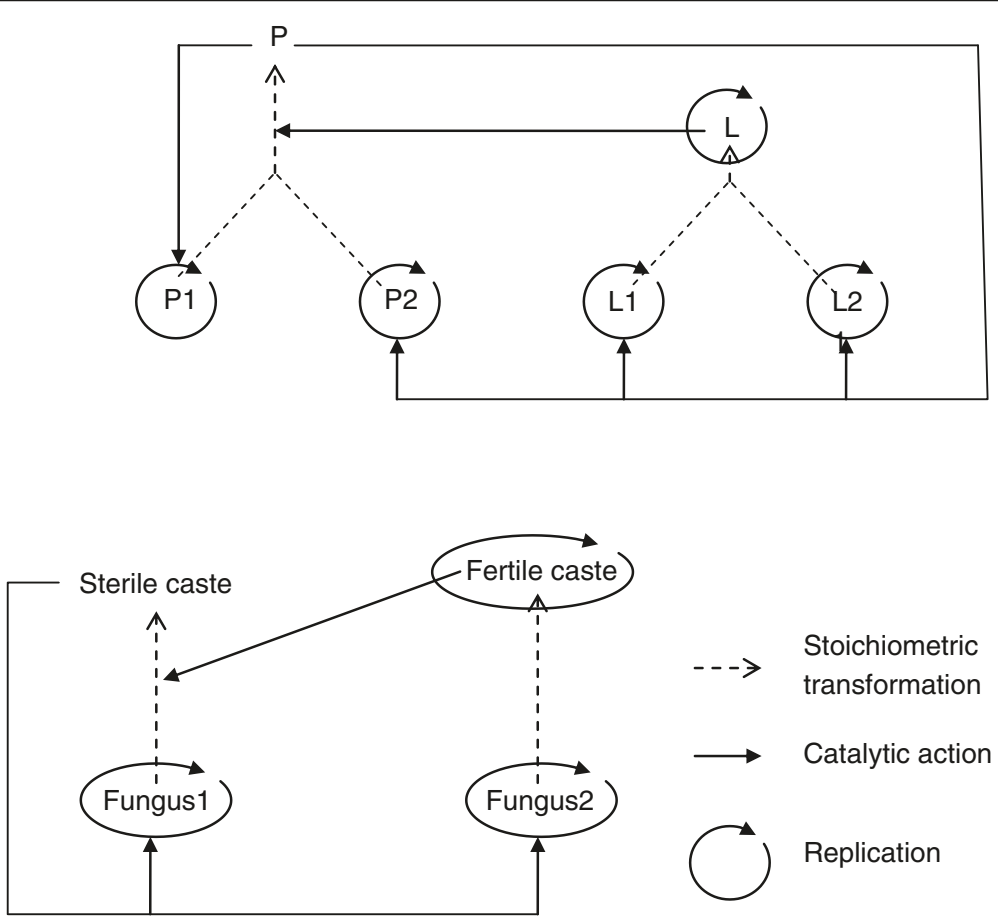

Figure $1 \mathrm{~A}$ non-hypercyclic molecular network [24] with multiple 'trophic levels' (top) and a possible biological analogue (bottom). P1 and P2: replicating oligomers as building blocks of polymerase P. L1 and L2: replicating oligomers as building blocks of ligase L. 
sense, or not? I think there isn't. First, with just the right food and ligase around, the polymerase will emerge: the latter is not a template, neither an informational replicator. Second, although it is true that $\mathrm{P}$ has a positive kinetic effect on itself as well as on $\mathrm{L}$, but $\mathrm{P}$, despite being a nucleic acid, is more analogous to protein enzymes (catalyzing metabolism) than genes. If metabolism is an autocatalytic network [25], then the analogy is very close. Metabolites are necessary for the replication of the genes and the formation of enzymes, catalysed by the genes. From a purely formalistic view [26] it is true that every catalyst contributing to the growth of a cell is an autocatalyst in the broad sense (it feeds back positively on its own growth), but the evolution of cooperation is a question related to the genetic/informational effects on the cooperative act $[8,25]$. Whereas it is meaningful to speak about genes cooperating in a cell, it is not revealing from the point of view of information and evolution to say that enzymes cooperate with genes, unless enzymes can informationally influence the spread of their variants, somehow transcending the genetic effects of the genes.

\section{Conclusion}

Systems chemistry has one of its intellectual roots in theoretical/evolutionary biology. Although this conceptually remarkably clear field has contributed to systems chemistry a lot [25], some chemists, mostly but not exclusively on the experimental side have proven to be surprisingly refractory to conceptual clarity. Experimental results are not in the void and, contrary to naïve presuppositions, they do not "speak for themselves": they are interpreted within a hypothetico-deductive framework. Attention should thus be given to conceptual issues, since category errors trigger one to look for supporting theory in the wrong file. Collectively autocatalytic systems without (at least) second-order catalysis have not much to do with hypercycles or cooperation sensu evolutionary biology beyond a vague resemblance on the grounds of a network and autocatalysis being involved. In particular, the importance of the genetic (informational) effect on the supposedly cooperative act is often overlooked. As in every field of science, we should aim at conceptual clarity also in systems chemistry. The sooner this happens, the better.

\section{Competing interests}

The author has no competing interests.

\section{Acknowledgements}

Financial support has been provided by the European Research Council under the European Community's Seventh Framework Programme (FP7/ 20072013)/ ERC grant agreement no [294332], EvoEvo project, and the Hungarian National Office for Research and Technology (NAP 2005/ KCKHA005). Comments by Dr. Mauro Santos are gratefully acknowledged.

\section{Author details}

${ }^{1}$ Parmenides Center for the Conceptual Foundations of Science, Kirchplatz 1 Pullach, Munich D-82049, Germany. ${ }^{2}$ Faculty of Biology, Ludwig Maximilians University Munich, Munich, Germany. ${ }^{3}$ Biological Insititute and Research Group in Evolutionary Ecology and Theoretical Biology, Eötvös University, Budapest, Hungary.

Received: 30 January 2013 Accepted: 8 February 2013

Published: 11 February 2013

\section{References}

1. Eigen M: Self-organization of matter and the evolution of biological macromolecules. Naturwissenschaften 1971, 58:465-523.

2. Eigen M, Schuster P: The hypercycle: A principle of natural self-organization. Berlin: Springer-Verlag; 1979.

3. Hofbauer J, Sigmund K: The theory of evolution and dynamical systems. Cambridge: Cambridge University Press; 1988.

4. Ricard J, Noat G: Electrostatic effects and the dynamics of enzyme reactions at the surface of plant cells 1 . A theory of the ionic control of a complex multi-enzyme system. Eur J Biochem 1986, 155:183-190.

5. Ricard J: Dynamics of multi-enzyme reactions, cell growth and perception of ionic signals from the external milieu. J Theor Bio/ 1987, 128:253-278.

6. Szathmáry E: A hypercyclic illusion. J Theor Biol 1988, 134:561-563.

7. Lee DH, Severin K, Yokobayashi Y, Ghadiri MR: Emergence of symbiosis in peptide self-replication through a hypercyclic network. Nature 1997, 390:591-594.

8. Nowak MA: Evolutionary Dynamics: Exploring the Equations of Life. Cambridge: The Belknap Press of Harvard University Press; 2006.

9. Kauffman S: Autocatalytic sets of proteins. J Theor Biol 1986, 119:1-24.

10. Sievers D, von Kiedrowski G: Self-replication of complementary nucleotide-based oligomers. Nature 1994, 369:221-224.

11. Sievers D, von Kiedrowski G: Self-replication of hexadeoxynucleotide analogues: autocatalysis versus cross-catalysis. Chem Eur J 1998, 4:629-641.

12. Szathmáry E: Evolution of replicators. Phil Trans R Soc Lond B 2000, 355:1669-1676.

13. Lee DH, Severin K, Yokobayashi Y, Ghadiri MR: Corrections: Emergence of symbiosis in peptide self-replication through a hypercyclic network. Nature 1998, 394:101.

14. Vaidya N, Manapat M, Chen IA, Xulvi-Brunet R, Hayden EJ, Lehman N: Spontaneous network formation among cooperative RNA replicators. Nature 2012, 491:72-77.

15. Attwater J, Holliger P: The cooperative gene. Nature 2012, 491:48-49.

16. Ellington AD: Origins for everyone. Evo Edu Outreach 2012, 5:361-366.

17. Greenwald J, Reik R: On the possible amyloid origin of protein folds. J Mol Biol 2012, 421:417-426.

18. Ellington AD: Back to the future of nucleic acid self-amplification. Nat Chem Biol 2009, 5:200-201.

19. Cousins GRL, Poulsen SA, Sanders JKM: Molecular evolution: dynamic combinatorial libraries, autocatalytic networks and the quest for molecular function. Curr Opin Chem Biol 2000, 4:270-279.

20. Beutel KM, Peacock-López E: Complex dynamics in a cross-catalytic selfreplication mechanism. J Chem Phys 2007, 126:125104.

21. Li X, Chmielewski J: Challenges in the design of self replicating peptides. Org Biomol Chem 2003, 1:901-904.

22. Issac R, Ham YW, Chmielewski J: The design of self-replicating helical peptides. Curr Opin Struct Biol 2001, 11:458-463.

23. Kauffman S, Ellington AD: Thinking combinatorially. Curr Opin Chem Biol 1999, 3:256-259.

24. Meyer AJ, Ellefson JW, Ellington AD: Abiotic self-replication. Acc Chem Res 2012, 45:2097-2105.

25. Maynard Smith J, Szathmáry E: The Major Transitions in Evolution. Oxford: Freeman; 1995.

26. Zachar I, Szathmáry E: A New Replicator: A theoretical framework for analysing replication. BMC Biol 2010, 8:21.

doi:10.1186/1759-2208-4-1

Cite this article as: Szathmáry: On the propagation of a conceptual error concerning hypercycles and cooperation. Journal of Systems Chemistry $20134: 1$. 